\title{
THE FIRST NFIL3/E4BP4 TRANSCRIPTION FACTOR WITH IMMUNE DEFENSE IDENTIFIED IN THE HONG KONG OYSTER, CRASSOSTREA HONGKONGENSIS
}

\author{
Jun Li, Ying Liu, Yuehuan Zhang, Yang Zhang, Fan Mao, Ziniu Yu* \\ Key Laboratory of Tropical Marine Bio-Resources and Ecology, South China Sea Institute of \\ Oceanology, Chinese Academy of Sciences, Guangzhou 510301, P. R. China
}

\begin{abstract}
Nuclear factor interleukin 3-regulated (NFIL3), also called E4 binding protein (E4BP4), was first identified as an adenovius E4 promotor and human IL3 promoter-binding protein. It is a basic leucine zipper (bZIP) type transcription factor and constitutively expressed in a variety of tissues, including spleen, prostate, testis, ovary, heart, brain, lung, liver, skeletal muscle, and intestine. In this study, we identified an NFIL3 gene (designated as ChNFIL3) in the Hong Kong oyster, Crassostrea hongkongensis. The full-length ChNFIL3 cDNA is $2221 \mathrm{bp}$ including 5' and 3'-untranslated regions (UTRs) of 303 and $382 \mathrm{bp}$, respectively, and an open reading frame (ORF) of $1536 \mathrm{bp}$. The gene encodes a 511 amino acid polypeptide with an estimated molecular mass of $57.4 \mathrm{kDa}$. SMART analysis shows that ChNFIL3 contains two typical bZIP domains. Phylogenetic tree revealed that ChNFIL3 was grouped into the clade of its invertebrate homologs. Tissue distribution analysis revealed a ubiquitous presence of ChNFIL3. The upregulated transcripts of ChNFIL3 in response to bacterial challenge suggest that ChNFIL3 is involved in innate immunity against pathogen infection. Recombinant ChNFIL3 protein can enhance the mRNA transcripts of inflammatory cytokines Interleukin-17 in vitro. ChNFIL3 is mainly localized to the nucleus in HEK293 cells by immunofluorescence microscopy. Moreover, luciferase reporter assays reveal that the over-expression of ChNFIL3 upregulates the transcriptional activities of NF- $\kappa$ B reporter gene in a dose-dependent manner. Our results presents the first experimental evidence of the presence and functional characteristics of NFIL3 in mollusks, which suggest that ChNFIL3 plays an essential role in innate defense in oysters.
\end{abstract}

Keywords: Crassostrea hongkongensis; NFIL3; NF-кB; Sub-cellular localization; immune defense

Corresponding author. Tel:+862089102507; Fax: 862089102507.

E-mail address: carlzyu@scsio.ac.cn (Z. Yu). 